\title{
A LIMIT THEOREM FOR DISCRETE GALTON-WATSON BRANCHING PROCESSES WITH IMMIGRATION
}

\author{
ZENGHU LI,* Beijing Normal University
}

\begin{abstract}
We provide a simple set of sufficient conditions for the weak convergence of discrete-time, discrete-state Galton-Watson branching processes with immigration to continuous-time, continuous-state branching processes with immigration.
\end{abstract}

Keywords: Markov chain; branching process with immigration; limit theorem; weak convergence; generating function

2000 Mathematics Subject Classification: Primary 60J80

\section{Introduction}

Let $(g, h)$ be a pair of probability generating functions. By a discrete-time, discrete-state Galton-Watson branching process with immigration (DBI process) corresponding to $(g, h)$ we mean a discrete-time Markov chain $\{y(n), n=0,1, \ldots\}$ with state space $\mathbb{N}:=\{0,1, \ldots\}$ and one-step transition matrix $P(i, j)$ defined by

$$
\sum_{j=0}^{\infty} P(i, j) z^{j}=g(z)^{i} h(z), \quad i=0,1, \ldots, \quad 0 \leq z \leq 1 .
$$

The intuitive meaning of the process is clear from (1.1). In particular, if $h(z) \equiv 1$, we simply call $\{y(n), n=0,1, \ldots\}$ a discrete-time, discrete-state Galton-Watson branching process (DB process).

Kawazu and Watanabe (1971) systematically studied the limit theorems of DBI processes and characterized the class of the limit processes completely as continuous-time, continuousstate branching processes with immigration (CBI processes). Let us consider a special class of the CBI processes introduced in Kawazu and Watanabe (1971). Suppose that $R$ is a function on $[0, \infty)$ defined by

$$
R(\lambda)=\beta \lambda-\alpha \lambda^{2}-\int_{0}^{\infty}\left(\mathrm{e}^{-\lambda u}-1+\frac{\lambda u}{1+u^{2}}\right) \mu(\mathrm{d} u),
$$

where $\beta \in \mathbb{R}$ and $\alpha \geq 0$ are constants and $\min \left\{1, u^{2}\right\} \mu(\mathrm{d} u)$ is a finite measure on $(0, \infty)$, and that $F$ is a function on $[0, \infty)$ defined by

$$
F(\lambda)=b \lambda+\int_{0}^{\infty}\left(1-\mathrm{e}^{-\lambda u}\right) m(\mathrm{~d} u)
$$

Received 8 December 2004; revision received 28 August 2005.

* Postal address: School of Mathematical Sciences, Beijing Normal University, Beijing, 100875, P. R. China.

Email address: lizh@bnu.edu.cn 
where $b \geq 0$ is a constant and $\min \{1, u\} m(\mathrm{~d} u)$ is a finite measure on $(0, \infty)$. A Markov process $\{y(t), t \geq 0\}$ with state space $\mathbb{R}_{+}:=[0, \infty)$ is called a CBI process if it has transition semigroup $\left(P_{t}\right)_{t \geq 0}$ given by

$$
\int_{0}^{\infty} \mathrm{e}^{-\lambda y} P_{t}(x, \mathrm{~d} y)=\exp \left\{-x \psi_{t}(\lambda)-\int_{0}^{t} F\left(\psi_{s}(\lambda)\right) \mathrm{d} s\right\}, \quad \lambda \geq 0,
$$

where $\psi_{t}(\lambda)$ is the unique solution of

$$
\frac{\mathrm{d} \psi_{t}}{\mathrm{~d} t}(\lambda)=R\left(\psi_{t}(\lambda)\right), \quad \psi_{0}(\lambda)=\lambda
$$

Clearly, the transition semigroup $\left(P_{t}\right)_{t \geq 0}$ defined by (1.3) is stochastically continuous. In particular, if $F(\lambda) \equiv 0$, then we simply call $\{y(t), t \geq 0\}$ a continuous-time, continuous-state branching process (CB process).

A CBI process is said to be conservative if it does not explode, that is, $\mathrm{P}_{x}\{y(t)<\infty\}=1$ for every $t \geq 0$ and $x \in \mathbb{R}_{+}$, where $\mathrm{P}_{x}$ denotes the conditional law given that $y(0)=x$. By Kawazu and Watanabe (1971, Theorem 1.2), the process is conservative if and only if

$$
\int_{0+} R^{*}(\lambda)^{-1} \mathrm{~d} \lambda=\infty
$$

where $R^{*}(\lambda)=\max \{0, R(\lambda)\}$. (This is a correction to Equation (1.21) of Kawazu and Watanabe (1971).)

Let $\left\{b_{k}\right\}$ and $\left\{c_{k}\right\}$ be sequences of positive numbers such that $b_{k} \rightarrow \infty$ and $c_{k} \rightarrow \infty$ as $k \rightarrow \infty$. Let $\left\{y_{k}(n), n \geq 0\right\}$ be a sequence of DBI processes corresponding to the parameters $\left\{\left(g_{k}, h_{k}\right)\right\}$ and assume that $y_{k}(0)=c_{k}$. Suppose that, for all $t \geq 0$ and $\lambda \geq 0$, the limits

$$
\lim _{k \rightarrow \infty} g_{k}^{[k t]}\left(\mathrm{e}^{-\lambda / b_{k}}\right)^{c_{k}}=: \phi_{1}(t, \lambda) \quad \text { and } \quad \lim _{k \rightarrow \infty} \prod_{j=0}^{[k t]-1} h_{k}\left(g_{k}^{j}\left(\mathrm{e}^{-\lambda / b_{k}}\right)\right)=: \phi_{2}(t, \lambda)
$$

exist and the convergence is locally uniform in $\lambda \geq 0$ for each fixed $t \geq 0$, where $g_{k}^{j}$ denotes the $j$-order composition of $g_{k}$ and $[k t]$ denotes the integer part of $k t$.

Theorem 1.1. (Kawazu and Watanabe (1971, Theorem 2.1).) Suppose that (1.5) holds and that $\phi_{1}(t, \lambda)<1$, for some $t>0$ and $\lambda>0$. Then $\left\{y_{k}([k t]) / b_{k}, t \geq 0\right\}$ converges in finitedimensional distributions to a stochastically continuous, conservative CBI process $\{y(t), t \geq 0\}$ with transition semigroup given by (1.3).

Based on this theorem, Kawazu and Watanabe (1971) showed that, given each stochastically continuous, conservative CBI process $\{y(t), t \geq 0\}$, there is a sequence of positive numbers $\left\{b_{k}\right\}$, where $b_{k} \rightarrow \infty$, and a sequence of DBI processes $\left\{y_{k}(n), n \geq 0\right\}$ such that $\left\{y_{k}([k t]) / b_{k}, t \geq 0\right\}$ converges in finite-dimensional distributions to $\{y(t), t \geq 0\}$. Their results have become the basis of many studies of branching processes with immigration; see, for example, Pitman and Yor (1982) and Shiga and Watanabe (1973). On the other hand, since (1.5) involves complicated compositions of the probability generating functions, it is sometimes not so easy to verify. In view of the characterizations (1.1), (1.3), and (1.4) of the two classes of process, we naturally expect some simple sufficient conditions for the convergence of the DBI processes to the CBI processes given in terms of the parameters $(g, h)$ and $(R, F)$. The purpose of this note is to provide a set of conditions of this type. For the convenience of proof, we 
shall discuss the convergence of $\left\{y_{k}\left(\left[\gamma_{k} t\right]\right) / k, t \geq 0\right\}$ for some sequence of positive numbers $\left\{\gamma_{k}\right\}$ with $\gamma_{k} \rightarrow \infty$, which is slightly different from the scaling of Kawazu and Watanabe (1971). Instead of the convergence of finite-dimensional distributions, we shall consider the weak convergence on the space of càdlàg functions $D\left([0, \infty), \mathbb{R}_{+}\right)$.

\section{The limit theorem}

In this section, we prove a limit theorem for DBI processes on the space $D\left([0, \infty), \mathbb{R}_{+}\right)$. Let $F$ be defined by (1.2). For simplicity we assume that the function $R$ is given by

$$
R(\lambda)=\beta \lambda-\alpha \lambda^{2}-\int_{0}^{\infty}\left(\mathrm{e}^{-\lambda u}-1+\lambda u\right) \mu(\mathrm{d} u), \quad \lambda \geq 0,
$$

where $\beta \in \mathbb{R}$ and $\alpha \geq 0$ are constants and $\min \left\{u, u^{2}\right\} \mu(\mathrm{d} u)$ is a finite measure on $(0, \infty)$. Suppose that $\{y(t), t \geq 0\}$ is a CBI process corresponding to $(R, F)$. Let $\left\{y_{k}(n), n \geq 0\right\}$ be a sequence of DBI processes corresponding to the parameters $\left\{\left(g_{k}, h_{k}\right)\right\}$ and let $\left\{\gamma_{k}\right\}$ be a sequence of positive numbers. For $0 \leq \lambda \leq k$, set

$$
F_{k}(\lambda)=\gamma_{k}\left[1-h_{k}\left(1-\frac{\lambda}{k}\right)\right]
$$

and

$$
R_{k}(\lambda)=k \gamma_{k}\left[\left(1-\frac{\lambda}{k}\right)-g_{k}\left(1-\frac{\lambda}{k}\right)\right] .
$$

Let us consider the following set of conditions.

(A) As $k \rightarrow \infty$, we have $\gamma_{k} \rightarrow \infty$ and $\gamma_{k} / k \rightarrow \gamma_{0}$, for some $\gamma_{0} \geq 0$.

(B) As $k \rightarrow \infty$, the sequence $\left\{F_{k}\right\}$ defined by (2.2) converges to a continuous function.

(C) The sequence $\left\{R_{k}\right\}$ defined by (2.3) is uniformly Lipschitz on each bounded interval, and converges to a continuous function as $k \rightarrow \infty$.

We remark that conditions (B) and (C) parallel the sufficient conditions for the convergence of continuous-time, discrete-state branching processes with immigration; see, for example, Li (1992) for discussion in the setting of measure-valued processes. Based the results of Li (1991), the following lemma can be proved by modifying the arguments of the proofs of Li (1992, Lemmas 3.4 and 4.1).

Lemma 2.1. (i) Under conditions (B) and (C), the limit functions $F$ and $R$ of $\left\{F_{k}\right\}$ and $\left\{R_{k}\right\}$ have representations (1.2) and (2.1), respectively.

(ii) For any $(F, R)$ given by (1.2) and (2.1), there are sequences $\left\{\gamma_{k}\right\}$ and $\left\{\left(g_{k}, h_{k}\right)\right\}$ as above such that conditions $(A),(B)$, and $(C)$ hold with $F_{k} \rightarrow F$ and $R_{k} \rightarrow R$.

For $\lambda \geq 0$ we set $S_{k}(\lambda)=k \gamma_{k}\left[(1-\lambda / k)-g_{k}\left(\mathrm{e}^{-\lambda / k}\right)\right]$.

Lemma 2.2. Under conditions $(A)$ and $(C)$, let $R=\lim _{k \rightarrow \infty} R_{k}$. Then we have

$$
\lim _{k \rightarrow \infty} S_{k}(\lambda)=R(\lambda)-\frac{\gamma_{0} \lambda^{2}}{2} \text { and } \lim _{k \rightarrow \infty} \gamma_{k}\left[1-g_{k}\left(\mathrm{e}^{-\lambda / k}\right)\right]=\gamma_{0} \lambda
$$

uniformly on each bounded interval. 
Proof. By the mean value theorem, we obtain

$$
S_{k}(\lambda)=R_{k}(\lambda)-k \gamma_{k} g_{k}^{\prime}\left(\eta_{k}\right)\left(\mathrm{e}^{-\lambda / k}-1+\frac{\lambda}{k}\right),
$$

where $1-\lambda / k<\eta_{k}<\mathrm{e}^{-\lambda / k}$ and $g_{k}^{\prime}$ denotes the derivative of $g_{k}$. Under condition (C), the sequence $R_{k}^{\prime}(\lambda)=\gamma_{k}\left[g_{k}^{\prime}(1-\lambda / k)-1\right]$ is uniformly bounded on each bounded interval $\lambda \in[0, l], l \geq 0$. Thus, $g_{k}^{\prime}(1-\lambda / k) \rightarrow 1$ uniformly on each bounded interval. In particular, we have $g_{k}^{\prime}\left(\eta_{k}\right) \rightarrow 1$, and the first equality in (2.4) follows from condition (A) and (2.5). The second equality in (2.4) follows by a similar argument.

Theorem 2.1. Suppose that conditions (A), (B), and (C) hold with $F=\lim _{k \rightarrow \infty} F_{k}$ and $R=$ $\lim _{k \rightarrow \infty} R_{k}$. If $y_{k}(0) / k$ converges in distribution to $y(0)$, then $\left\{y_{k}\left(\left[\gamma_{k} t\right]\right) / k, t \geq 0\right\}$ converges in distribution on $D\left([0, \infty), \mathbb{R}_{+}\right)$to the CBI process $\{y(t), t \geq 0\}$ corresponding to $(R, F)$ with initial value $y(0)$.

Proof. Let $\left(P_{t}\right)_{t \geq 0}$ denote the transition semigroup of the CBI process corresponding to $(R, F)$. For $\lambda>0$ and $x \geq 0$, set $e_{\lambda}(x)=\mathrm{e}^{-\lambda x}$. We denote by $D_{1}$ the linear hull of $\left\{e_{\lambda}, \lambda>0\right\}$. Then $D_{1}$ is an algebra which strongly separates the points of $\mathbb{R}_{+}$. Let $C_{0}\left(\mathbb{R}_{+}\right)$be the space of continuous functions on $\mathbb{R}_{+}$vanishing at infinity. By the Stone-Weierstrass theorem, $D_{1}$ is dense in $C_{0}\left(\mathbb{R}_{+}\right)$for the supremum norm; see, for example, Hewitt and Stromberg (1965, pp. 98-99). For $\lambda>0$ we set

$$
A e_{\lambda}(x)=-\mathrm{e}^{-\lambda x}[x R(\lambda)+F(\lambda)], \quad x \in \mathbb{R}_{+},
$$

and extend the definition of $A$ to $D_{1}$ by linearity. Then $A:=\left\{(f, A f), f \in D_{1}\right\}$ is a linear subspace of $C_{0}\left(\mathbb{R}_{+}\right) \times C_{0}\left(\mathbb{R}_{+}\right)$. Since $D_{1}$ is invariant under $\left(P_{t}\right)_{t \geq 0}$, it is a core of $A$; see, for example, Ethier and Kurtz (1986, p. 17). In other words, the semigroup $\left(P_{t}\right)_{t \geq 0}$ is generated by the closure of $A$; see, for example, Ethier and Kurtz (1986, p. 15 and p. 17). Note that $\left\{y_{k}(n) / k, n \geq 0\right\}$ is a Markov chain with state space $E_{k}:=\{0,1 / k, 2 / k, \ldots\}$ and one-step transition probability $Q_{k}(x, \mathrm{~d} y)$ determined by

$$
\int_{E_{k}} \mathrm{e}^{-\lambda y} Q_{k}(x, \mathrm{~d} y)=g_{k}\left(\mathrm{e}^{-\lambda / k}\right)^{k x} h_{k}\left(\mathrm{e}^{-\lambda / k}\right) .
$$

Then the (discrete) generator $A_{k}$ of $\left\{y_{k}\left(\left[\gamma_{k} t\right]\right) / k, t \geq 0\right\}$ is given by

$$
\begin{aligned}
A_{k} e_{\lambda}(x) & =\gamma_{k}\left[g_{k}\left(\mathrm{e}^{-\lambda / k}\right)^{k x} h_{k}\left(\mathrm{e}^{-\lambda / k}\right)-\mathrm{e}^{-\lambda x}\right] \\
& =\gamma_{k}\left[\exp \left\{x k \alpha_{k}(\lambda)\left(g_{k}\left(\mathrm{e}^{-\lambda / k}\right)-1\right)\right\} \exp \left\{\beta_{k}(\lambda)\left(h_{k}\left(\mathrm{e}^{-\lambda / k}\right)-1\right)\right\}-\mathrm{e}^{-\lambda x}\right],
\end{aligned}
$$

where

$$
\alpha_{k}(\lambda)=\left(g_{k}\left(\mathrm{e}^{-\lambda / k}\right)-1\right)^{-1} \log g_{k}\left(\mathrm{e}^{-\lambda / k}\right)
$$

and $\beta_{k}(\lambda)$ is defined by the same formula with $g_{k}$ replaced by $h_{k}$. Under conditions (A), (B), and $(C)$, it is easy to show that

$$
\lim _{k \rightarrow \infty}\left(g_{k}\left(\mathrm{e}^{-\lambda / k}\right)-1\right)=\lim _{k \rightarrow \infty}\left(h_{k}\left(\mathrm{e}^{-\lambda / k}\right)-1\right)=0
$$

and

$$
\lim _{k \rightarrow \infty} \alpha_{k}(\lambda)=\lim _{k \rightarrow \infty} \beta_{k}(\lambda)=1
$$


Then we have

$$
A_{k} e_{\lambda}(x)=-\mathrm{e}^{-\lambda x}\left[x \alpha_{k}(\lambda) S_{k}(\lambda)+x \gamma_{k}\left(\alpha_{k}(\lambda)-1\right) \lambda+H_{k}(\lambda)\right]+o(1),
$$

where

$$
H_{k}(\lambda)=\gamma_{k} \beta_{k}(\lambda)\left(1-h_{k}\left(\mathrm{e}^{-\lambda / k}\right)\right) .
$$

By elementary calculations we find that

$$
\alpha_{k}(\lambda)=1+\frac{1}{2}\left(1-g_{k}\left(\mathrm{e}^{-\lambda / k}\right)\right)+o\left(1-g_{k}\left(\mathrm{e}^{-\lambda / k}\right)\right),
$$

and so $\lim _{k \rightarrow \infty} \gamma_{k}\left(\alpha_{k}(\lambda)-1\right)=\gamma_{0} \lambda / 2$ by Lemma 2 .2. It follows that

$$
\lim _{k \rightarrow \infty}\left[\alpha_{k}(\lambda) S_{k}(\lambda)+\gamma_{k}\left(\alpha_{k}(\lambda)-1\right) \lambda\right]=R(\lambda) .
$$

By the argument of the proof of Lemma 2.2, we can show that

$$
\lim _{k \rightarrow \infty} H_{k}(\lambda)=\lim _{k \rightarrow \infty} F_{k}(\lambda)=F(\lambda) .
$$

In view of (2.6) and (2.7), we obtain

$$
\lim _{k \rightarrow \infty} \sup _{x \in E_{k}}\left|A_{k} e_{\lambda}(x)-A e_{\lambda}(x)\right|=0,
$$

for each $\lambda>0$. This clearly implies that

$$
\lim _{k \rightarrow \infty} \sup _{x \in E_{k}}\left|A_{k} f(x)-A f(x)\right|=0,
$$

for each $f \in D_{1}$. From Ethier and Kurtz (1986, p. 226 and pp. 233-234) we find that $\left\{y_{k}\left(\left[\gamma_{k} t\right]\right) / k, t \geq 0\right\}$ converges in distribution on $D\left([0, \infty), \mathbb{R}_{+}\right)$to the CBI process corresponding to $(R, F)$.

By Lemma 2.1 and Theorem 2.1, for any functions $(R, F)$ given by (1.2) and (2.1), there is a sequence of positive numbers $\left\{\gamma_{k}\right\}$ and a sequence of DBI processes $\left\{y_{k}(n), n \geq 0\right\}$ such that $\left\{y_{k}\left(\left[\gamma_{k} t\right]\right) / k, t \geq 0\right\}$ converges in distribution on $D\left([0, \infty), \mathbb{R}_{+}\right)$to the CBI process corresponding to $(R, F)$.

\section{Generalized Ray-Knight theorems}

As an example of the applications of their limit theorems, Kawazu and Watanabe (1971) re-proved the Ray-Knight theorems of diffusion characterizations of the Brownian local time. In this section, we generalize the results to the case of a Brownian motion with drift. We refer the reader to Le Gall and Le Jan (1998) for another adequate formulation of the Ray-Knight theorems for general Lévy processes.

Let $A=\alpha \mathrm{d}^{2} / \mathrm{d} x^{2}+\beta \mathrm{d} / \mathrm{d} x$ for given constants $\alpha>0$ and $\beta \in \mathbb{R}$. Then $A$ generates a one-dimensional Brownian motion with drift $\left(X_{t}, \mathcal{F}_{t}, \mathrm{P}_{x}\right)$. The local time of $\left\{X_{t}, t \geq 0\right\}$ is a continuous two-parameter process $\{l(t, x), t \geq 0, x \in \mathbb{R}\}$ such that the following property holds almost surely:

$$
2 \int_{B} l(s, x) \mathrm{d} x=\int_{0}^{t} \mathbf{1}_{B}\left(X_{s}\right) \mathrm{d} s, \quad B \in \mathscr{B}(\mathbb{R}) .
$$

Here $\mathscr{B}(\mathbb{R})$ denotes the Borel $\sigma$-algebra of $\mathbb{R}$ and $\mathbf{1}_{B}$ is the indicator function of $B$. For a fixed $a \geq 0$, let

$$
l^{-1}(u, a)=\inf \{t \geq 0: l(t, a)=u\} .
$$


Theorem 3.1. The process

$$
\xi_{u}(t)=l\left(l^{-1}(u, a), a+t\right), \quad t \geq 0,
$$

is a diffusion generated by

$$
x \frac{\mathrm{d}^{2}}{\mathrm{~d} x^{2}}+\frac{\beta}{\alpha} x \frac{\mathrm{d}}{\mathrm{d} x} .
$$

Proof. We follow the ideas of Kawazu and Watanabe (1971, Example 2.2). For $c \in \mathbb{R}$ let $\sigma_{c}=\inf \left\{t \geq 0: X_{t}=c\right\}$. Let $\delta>0$ and let $u_{\delta}(x)=\mathrm{P}_{x}\left\{\sigma_{\delta}<\sigma_{-\delta}\right\}=1-\mathrm{P}_{x}\left\{\sigma_{\delta}>\sigma_{-\delta}\right\}$. Then $u_{\delta}(\cdot)$ satisfies

$$
\alpha \frac{\mathrm{d}^{2}}{\mathrm{~d} x^{2}} u_{\delta}(x)+\beta \frac{\mathrm{d}}{\mathrm{d} x} u_{\delta}(x)=0, \quad|x| \leq \delta,
$$

with $u_{\delta}(\delta)=1$ and $u_{\delta}(-\delta)=0$. Solving this boundary value problem, we find that

$$
u_{\delta}(x)=\frac{\exp \{\beta \delta / \alpha\}-\exp \{-\beta x / \alpha\}}{\exp \{\beta \delta / \alpha\}-\exp \{-\beta \delta / \alpha\}} .
$$

By a $\delta$-downcrossing at $x \in \mathbb{R}$ before time $T>0$ we mean an interval $[u, v] \subset[0, T)$ such that $X_{u}=x+\delta, X_{v}=x$, and $x<X_{t}<x+\delta$ for all $u<t<v$. Let $\eta_{\delta}$ denote the number of $\delta$-downcrossings at 0 before time $\sigma_{-\delta}$. By the property of independent increments of the Brownian motion with drift, we have

$$
\mathrm{E}_{0}\left[z^{\eta_{\delta}}\right]=\sum_{i=0}^{\infty}(1-p)(p z)^{i}=\frac{q}{1-p z},
$$

where $p=u_{\delta}(0), q=1-p$, and $\mathrm{E}_{0}$ denotes the expectation under $\mathrm{P}_{0}$. Let $x_{i}=a+i / k$ for $i \geq 0$ and $k \geq 1$ and let $Z_{k}(i)$ denote the number of $(1 / k)$-downcrossings at $x_{i}$ before time $l^{-1}(u, a)$. It is easy to see that $Z_{k}(i+1)$ is the sum of $Z_{k}(i)$ independent copies of $\eta_{1 / k}$. Thus, $\left\{Z_{k}(i), i=0,1, \ldots\right\}$ is a DB process corresponding to the generating function

$$
g_{k}(z)=\frac{q_{k}}{1-p_{k} z}
$$

where $p_{k}=u_{1 / k}(0)$ and $q_{k}=1-p_{k}$. By a standard result for local times of diffusion processes, we obtain

$$
\lim _{k \rightarrow 0} \frac{Z_{1 / k}([k t])}{k}=l\left(l^{-1}(u, a), a+t\right)=\xi_{u}(t) ;
$$

see Itô and McKean (1965, p. 48 and p. 222). Then Theorem 2.1 implies that the limit $\left\{\xi_{u}(t), t \geq 0\right\}$ is a $\mathrm{CB}$ process corresponding to

$$
R(\lambda)=\lim _{k \rightarrow \infty} k^{2}\left[\left(1-\frac{\lambda}{k}\right)-g_{k}\left(1-\frac{\lambda}{k}\right)\right]=\frac{\beta}{\alpha} \lambda-\lambda^{2} .
$$

This proves the desired result.

Kawazu and Watanabe (1971, Theorem 2.3 and Example 2.2) proved the result of Theorem 3.1 in the special case $\beta=0$. In that case the generating function $g_{k}$ is actually independent of $k \geq 1$. In the general case, it seems difficult to check condition (1.5) for the sequence $\left\{g_{k}\right\}$. By arguments similar to those used above, we obtain the following theorem. 
Theorem 3.2. The process

$$
\eta_{u}(t)=l\left(l^{-1}(u, a), a-t\right), \quad 0 \leq t \leq a,
$$

is a diffusion generated by

$$
x \frac{\mathrm{d}^{2}}{\mathrm{~d} x^{2}}+\frac{\beta}{\alpha} x \frac{\mathrm{d}}{\mathrm{d} x}+\frac{\mathrm{d}}{\mathrm{d} x} .
$$

\section{Acknowledgements}

This research was supported by the NSFC, grant numbers 10121101 and 10525103 . I would like to thank the referee for a number of helpful comments.

\section{References}

Ethier, S. N. And Kurtz, T. G. (1986). Markov Processes: Characterization and Convergence. John Wiley, New York.

Hewitt, E. And Stromberg, K. (1965). Real and Abstract Analysis. Springer, Berlin.

Itô, K. And McKean, H. P., JR. (1965). Diffusion Processes and their Sample Paths. Springer, Berlin.

KaWAZU, K. AND WatanaBe, S. (1971). Branching processes with immigration and related limit theorems. Theory Prob. Appl. 16, 36-54.

Le Gall, J.-F. And Le Jan, Y. (1998). Branching processes in Lévy processes: the exploration process. Ann. Prob. 26, 213-252.

LI, Z. H. (1991). Integral representations of continuous functions. Chinese Sci. Bull. 36, 979-983.

LI, Z. H. (1992). Measure-valued branching processes with immigration. Stoch. Process. Appl. 43, $249-264$.

Pitman, J. AND Yor, M. (1982). A decomposition of Bessel bridges. Z. Wahrscheinlichkeitsth. 59, 425-457.

Shiga, T. AND WatANABE, S. (1973). Bessel diffusions as a one-parameter family of diffusion processes. Z. Wahrscheinlichkeitsth. 27, 37-46. 